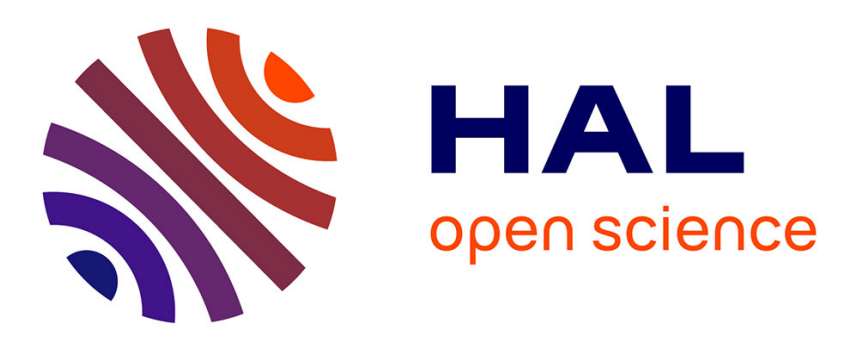

\title{
Construction and analysis of an adapted spectral finite element method to convective acoustic equations
} Andreas Hüppe, Gary Cohen, Sebastien Imperiale, Manfred Kaltenbacher

\section{To cite this version:}

Andreas Hüppe, Gary Cohen, Sebastien Imperiale, Manfred Kaltenbacher. Construction and analysis of an adapted spectral finite element method to convective acoustic equations. Communications in Computational Physics, 2016, 10.4208/cicp.250515.161115a . hal-01363149

\section{HAL Id: hal-01363149 \\ https://inria.hal.science/hal-01363149}

Submitted on 9 Sep 2016

HAL is a multi-disciplinary open access archive for the deposit and dissemination of scientific research documents, whether they are published or not. The documents may come from teaching and research institutions in France or abroad, or from public or private research centers.
L'archive ouverte pluridisciplinaire HAL, est destinée au dépôt et à la diffusion de documents scientifiques de niveau recherche, publiés ou non, émanant des établissements d'enseignement et de recherche français ou étrangers, des laboratoires publics ou privés. 


\title{
Construction and analysis of an adapted spectral finite element method to convective acoustic equations
}

\author{
Andreas Hüppe $^{\mathrm{a}}$, Gary Cohen ${ }^{\mathrm{b}}$, Sébastien Imperiale ${ }^{\mathrm{c}}$, Manfred Kaltenbacher ${ }^{\mathrm{a}}$ \\ ${ }^{a}$ TU Wien, Institute of Mechanics and Mechatronics, Getreidemarkt 9/A4, 1060 Wien, \\ Austria \\ ${ }^{b}$ Inria-CNRS-ENSTA, Saclay Ile-de-France, 91120 Palaiseau, France \\ ${ }^{c}$ Inria, Saclay Ile-de-France, 91120 Palaiseau, France
}

\begin{abstract}
The paper addresses the construction of a non spurious mixed spectral finite element (FE) method to problems in the field of computational aeroacoustics. Based on a computational scheme for the conservation equations of linear acoustics, the extension towards convected wave propagation is investigated. In aeroacoustic applications, the mean flow effects can have a significant impact on the generated sound field even for smaller Mach numbers. For those convective terms, the initial spectral FE discretization leads to non-physical, spurious solutions. Therefore, a regularization procedure is proposed and qualitatively investigated by means of discrete eigenvalues analysis of the discrete operator in space. A study of convergence and an application of the proposed scheme to simulate the flow induced sound generation in the process of human phonation underlines stability and validity.
\end{abstract}

Keywords: Spectral Finite Elements, Aeroacoustics, Perturbation Equations

\section{Introduction}

Constructing a numerical scheme to compute the acoustic field in flowing media is not an easy task. The first step is the choice of an appropriate physical model. The simplest one towards aeroacoustics is Lighthill's analogy [1], whose main drawback is that it does not separate flow and acoustic quantities and so the computed fluctuating pressure just converges to the acoustic one for observation points far away from the turbulent source region, where the mean flow velocity becomes zero. For acoustic propagation in the presence of a (non-uniform) mean flow or compressible, non-isentropic media, formulations based on the linearized Euler equations (LEE) [2] and acoustic perturbation equations [3, 4, 5] seem to be the most realistic models. From the mathematical

Email addresses: andreas.hueppe@tuwien.ac.at (Andreas Hüppe),

Gary.Cohen@ensta-paristech.fr (Gary Cohen), sebastien.imperiale@inria.fr (Sébastien Imperiale), manfred.kaltenbacher@tuwien.ac.at (Manfred Kaltenbacher) 
point of view, these models present an important problem, because the involved differential operators do not correspond to any classical functional space, and therefore no classical finite element fit to its numerical solution. Actually, the use of classical finite element approximations (in $H^{1}$ or $H($ div)) generates substantial parasitic waves produced by numerical spurious modes. The discontinuous Galerkin methods seem well adapted for the approximation of linearized Euler equations since they do not require any regularity of the solution. On the other hand, these methods are able to suppress parasitic waves by the use of a dissipative jump [6]. First implemented for triangular and tetrahedral meshes for these equations [7, 8, 9, these methods were then adapted to quadrilateral and hexahedral meshes using mass-lumped spectral elements [10. Another way to get a spurious free solution for a continuous finite element approximation is to get a coercive operator by adding a differential penalty term. This method, introduced by 11 for the Maxwell's equations, was applied to the time-harmonic Galbrun's equations [12] (which are equivalent to LEE) to which a penalty term using a curl-operator was added to get coercivity. However, this approach implies the numerical treatment of an additional operator, which substantially increases the computational time, in particular in time domain. In this paper, we present a finite element approximation in which the acoustic pressure is sought in $H^{1}$ and the irrotational part of the fluctuating velocity (acoustic particle velocity) in $\left(L^{2}\right)^{d}, d=2,3$. The discontinuous character of the second variable enables us to add a jump term which, as for Maxwell's equations [13, gets rid of spurious modes. This approach leads to a method based on continuous finite elements which is spurious free for a small additional cost.

After a brief presentation in section 2 of the acoustic perturbation equations we present in section 3 a straightforward extension of the mixed spectral FE to these equations. We illustrate and explain why such straightforward derivation is unstable. In section 4 we construct and study by a discrete eigenvalue analysis a stabilization and penalization procedures to rectify the previously proposed formulation. Finally in section 5 we present a numerical convergence analysis and a successful extension of the proposed scheme to three dimensional setups and spatially varying mean flows by means of a numerical computation of the aeroacoustic sound generated in the human phonation process.

\section{Acoustic Perturbation Equations}

A known system of perturbation equations for aeroacoustic computations based upon incompressible flow data is APE-1 as proposed by R. Ewert, which is a system of two PDEs (Eq. (47)-(48) in [3]). Assuming constant temperature and small Mach number, in the same article, the APE-2 variant is obtained. It results in PDEs written for the acoustic pressure $p$ and for the acoustic particle velocity $\mathbf{u}$ (irrotational part of the fluctuating velocity)

$$
\begin{aligned}
\frac{\partial p}{\partial t}+\rho_{0} c_{0}^{2} \nabla \cdot \mathbf{u}+\nabla \cdot\left(p \mathbf{u}_{0}\right) & =f, \\
\frac{\partial \mathbf{u}}{\partial t}+\left(\mathbf{u}_{0} \cdot \nabla\right) \mathbf{u}+\frac{\nabla p}{\rho_{0}} & =\mathbf{0},
\end{aligned}
$$


where $f$ denotes the source term. In the previous equations, $\rho_{0}$ and $c_{0}$ are assumed constant and positive, and denote the mean density and the speed of sound, respectively. The vector $\mathbf{u}_{0}$ describes the mean flow velocity, and is assumed to be constant. Furthermore, our forthcoming analysis still holds for a space varying and divergence free mean flow velocity or a piecewise constant mean flow velocity.

In the special case of a vanishing mean flow, i.e. $\mathbf{u}_{0}=\mathbf{0}$, we directly obtain the conservation equations of linear acoustics given in homogeneous form by

$$
\begin{aligned}
\frac{1}{c_{0}^{2} \rho_{0}} \frac{\partial p}{\partial t}+\nabla \cdot \mathbf{u} & =0, \\
\rho_{0} \frac{\partial \mathbf{u}}{\partial t}+\nabla p & =\mathbf{0} .
\end{aligned}
$$

This system can be transformed to obtain the acoustic wave equation. For the derivation we differentiate (3) with respect to time and take the divergence of (4). Subtracting both equations yields

$$
\frac{1}{c_{0}^{2}} \frac{\partial^{2} p}{\partial t^{2}}-\Delta p=0 .
$$

For the case of an arbitrary, non-vanishing mean-flow, this simplification cannot be done and a computational scheme must feature stable solutions for the system (1) and (2). In literature, most formulations for these perturbation equations are based upon finite difference (FD) or discontinuous Galerkin (DG) schemes (see e.g. 3, 8, 14]). Whereas FD schemes are known to be inflexible with respect to discretization, the number of degrees of freedom is highly increased within DG methods due to their element-local definition of unknowns. A stable finite element (FE) scheme with a reduced number of unknowns could thereby be an attractive alternative.

Even for the case of vanishing mean flow, i.e. the conservation equations (3) and (4), it is not trivial to obtain a stable finite element formulation due to the violation of the Ladyzhenskaya-Babuška-Brezzi (or inf-sup) condition for an associated elliptic problem (see [15, 16]). One possibility to obtain a stable solution is the utilization of a mixed formulation in which the unknowns are defined in different Sobolev spaces [17. A well posed mixed formulation was introduced in [18] and has already been investigated and extended by the authors [19, 20]. Especially regarding computational costs, this formulation has significant advantages when combined with a spectral finite element method which yields diagonal mass matrices.

\section{Mixed variational formulation of the Acoustic Perturbation Equa- tions}

Let $L^{2}$ be the space of Lebesque square integrable functions in the propagation domain $\Omega, H^{1}$ the Sobolev space of functions whose first derivatives are also in $L^{2}$ and $H(\mathbf{d i v})$ the space of functions whose divergence is in $L^{2}$. There 
are two stable combinations available, which fulfill the LBB condition at the continuous level in the case of vanishing mean flow:

1. Choose $p \in L^{2}$ and $\mathbf{u} \in H($ div $)$.

2. Choose $p \in H^{1}$ and $\mathbf{u} \in\left(L^{2}\right)^{d}$.

The first choice could be realized by using Raviart-Thomas elements 21]. The second choice can be realized using spectral finite elements 18 , which will be used here.

For the conservation equations of linear acoustics, the variational form is obtained by multiplication of (3) by a scalar test function $\varphi \in H^{1}$ and (4) with a vectorial test function $\psi \in\left(L^{2}\right)^{d}$. Integration over the computational domain $\Omega$ with boundary $\Gamma$ leads to

$$
\begin{aligned}
\frac{1}{\rho_{0} c_{0}^{2}} \int_{\Omega} \frac{\partial p}{\partial t} \varphi \mathbf{d} \Omega+\int_{\Omega} \nabla \varphi \cdot \mathbf{u} \mathbf{d} \Omega-\int_{\Gamma} \varphi \mathbf{u} \cdot \mathbf{n} \mathbf{d} \Gamma & =\int_{\Omega} f \varphi \mathbf{d} \Omega \\
\rho_{0} \int_{\Omega} \frac{\partial \mathbf{u}}{\partial t} \cdot \boldsymbol{\psi} \mathbf{d} \Omega+\int_{\Omega} \nabla p \cdot \boldsymbol{\psi} \mathbf{d} \Omega & =0 .
\end{aligned}
$$

To define the discrete functional spaces, we assume a domain, discretized by $N$ quadrilateral or hexahedral finite elements $K, \Omega=\cup_{j=1}^{N} K_{j}$. Furthermore, there is a bijective mapping $F_{j}$ to transform the grid element $K_{j}$ to the reference square or cubic element $\hat{K}$. Then the spaces of approximation are defined as

$$
\begin{aligned}
p_{h}, \varphi_{h} \in U_{h}^{k} & =\left\{q \in H^{1}|q|_{K_{j}} \circ F_{j} \in Q^{k}(\hat{K})\right\} \\
\mathbf{u}_{h}, \boldsymbol{\psi}_{h} \in V_{h}^{k} & =\left\{\mathbf{w} \in\left[L^{2}\right]^{d}\left|\frac{1}{\left|\mathcal{J}_{j}\right|} \mathcal{J}_{j} \mathbf{w}\right|_{K_{j}} \circ F_{j} \in\left[Q^{k}(\hat{K})\right]^{d}\right\} .
\end{aligned}
$$

In (8) and (9), $\mathcal{J}_{j}$ denotes the Jacobian matrix of the element mapping $F_{j},\left|\mathcal{J}_{j}\right|$ its determinant and $Q^{k}(\hat{K})$ the tensor product set of Lagrange polynomials of degree $k$. Thereby the acoustic particle velocity $\mathbf{u}$ is approximated discontinuously from element to element which is similar to a DG formulation.

First, it is obvious that the mixed formulation reduces the number of unknowns with respect to a DG formulation due to the continuous approximation of the pressure unknowns. Additionally, this formulation has the advantage, that the following relation holds when evaluating the integral over an arbitrary element $K_{j}$

$$
\int_{K_{j}} \nabla \varphi_{h} \cdot \boldsymbol{\psi}_{h} \mathbf{d} K_{j}=\int_{\hat{K}} \hat{\nabla} \hat{\varphi}_{h} \cdot \hat{\boldsymbol{\psi}}_{h} \mathbf{d} \hat{K}
$$

which provide, after using Gauss-Lobatto points for quadrature, sparse local matrices independent of the elements geometry. This enables very efficient implementations. In general the resulting semidiscrete system of equations takes the form

$$
\left(\begin{array}{cc}
\mathbf{D} & \mathbf{0} \\
\mathbf{0} & \mathbf{B}
\end{array}\right)\left(\begin{array}{l}
\dot{p_{h}} \\
\dot{\mathbf{u}}_{h}
\end{array}\right)+\left(\begin{array}{cc}
\mathbf{0} & -\mathbf{R} \\
\mathbf{R}^{T} & \mathbf{0}
\end{array}\right)\left(\begin{array}{l}
p_{h} \\
\mathbf{u}_{h}
\end{array}\right)=\left(\begin{array}{c}
\mathbf{F} \\
\mathbf{0}
\end{array}\right)
$$


Because of the utilized spectral element approximation, the matrix $\mathbf{D}$ is diagonal and the matrix $\mathbf{B}$ is $d \times d$ block-diagonal with $d=\{2,3\}$ being the dimension of the setup. As $\mathbf{D}$ and $\mathbf{B}$ are in addition symmetric positive definite, they can be inverted easily thus being well suited for the application of an explicit time stepping scheme. As already indicated in $(10)$, the matrix $\mathbf{R}$ is independent of the elements geometry and its structure only depends on the connectivity of the elements in the computational grid and is very sparse.

We notice that the only differences between the conservation equations (3) and (4) and the system of perturbation equations (1) and (2), are the convective terms $A$ and $B$ which are given in $\sqrt{12}$ and $\sqrt{13})$. Those terms are usually handled within finite element methods by employing some kind of upwinding schemes such as the Streamline-Upwind-Petrov-Galerkin (SUPG) approach as done e.g. for the Navier-Stokes equations in 22. These schemes alter the ansatz functions and increase the computational effort. Therefore, motivated by the good performance properties of the mixed spectral method, we intend to solve this system of equations using the same mixed variational ansatz as utilized for the conservation equation of linear acoustics.

For a shorter notation we neglect the source term. The variational form of (3), (4) for $\mathbf{u}, \boldsymbol{\psi}$ and $p, \varphi$ regular enough is given as

$$
\begin{gathered}
\int_{\Omega} \frac{\partial p}{\partial t} \varphi \mathrm{d} \Omega-\rho_{0} c_{0}^{2} \int_{\Omega} \mathbf{u} \cdot \nabla \varphi \mathrm{d} \Omega+\underbrace{\int_{\Omega}\left(\mathbf{u}_{0} \cdot \nabla p\right) \varphi \mathrm{d} \Omega}_{A}=0 \\
\int_{\Omega} \frac{\partial \mathbf{u}}{\partial t} \cdot \boldsymbol{\psi} \mathrm{d} \Omega+\underbrace{\int_{\Omega}\left(\mathbf{u}_{0} \cdot \nabla\right) \mathbf{u} \cdot \boldsymbol{\psi} \mathrm{d} \Omega}_{B}+\frac{1}{\rho_{0}} \int_{\Omega} \nabla p \cdot \boldsymbol{\psi} \mathrm{d} \Omega=0
\end{gathered}
$$

subjected to either Neumann boundary conditions (sound-hard wall) on $\Gamma_{N}$ and Dirichlet boundary conditions (sound-soft wall) on $\Gamma_{D}\left(\Gamma=\Gamma_{D} \cup \Gamma_{N}\right.$ and $\left.\Gamma_{D} \cap \Gamma_{N}=\emptyset\right)$. These conditions read

$$
\begin{cases}p=0, & \text { on } \Gamma_{D} \\ \mathbf{u}_{0} \cdot \mathbf{n}=\mathbf{u} \cdot \mathbf{n}=0, & \text { on } \Gamma_{N} .\end{cases}
$$

Having chosen regular enough test functions (with $\varphi$ vanishing on $\Gamma_{D}$ ), the term B satisfies

$$
B=\sum_{K_{i}} \int_{K_{i}}\left(\mathbf{u}_{0} \cdot \nabla\right) \mathbf{u} \cdot \boldsymbol{\psi} \mathrm{d} K .
$$

A tempting but wrong strategy to extend the discretization presented above in order to take into account convective terms is to use relation $[15)$ with $(\mathbf{u}, \boldsymbol{\psi}) \in$ $V_{h}^{k} \times V_{h}^{k}$.

Written in matrix notation we compute two additional matrices, $\mathbf{C}_{\mathbf{p}}$ and $\mathbf{C}_{\mathbf{u}}$, which result from the discretization of the bilinear forms $A$ and $B$.

$$
\underbrace{\left(\begin{array}{cc}
\mathbf{D} & \mathbf{0} \\
\mathbf{0} & \mathbf{B}
\end{array}\right)}_{\mathbf{M}}\left(\begin{array}{l}
\dot{p_{h}} \\
\dot{\mathbf{u}}_{h}
\end{array}\right)+\underbrace{\left(\begin{array}{cc}
\mathbf{C}_{\mathbf{p}} & -\mathbf{R} \\
\mathbf{R}^{T} & \mathbf{C}_{\mathbf{u}}
\end{array}\right)}_{\mathbf{K}}\left(\begin{array}{l}
p_{h} \\
\mathbf{u}_{h}
\end{array}\right)=\left(\begin{array}{c}
\mathbf{F} \\
\mathbf{0}
\end{array}\right) \text {. }
$$


Two major difficulties in the mixed finite element formulation appear:

1. The choice of the discontinuous space $L^{2}$ for the acoustic particle velocity is in contradiction with the simplified form of $B$ given by 15 .

2. Even for a spatially constant but arbitrarily directed mean flow field, the additional matrices may be asymmetric thus violating the evident skew symmetric structure of the discrete system observed in (11). This may give rise to eigenvalues with positive real part and thereby unstable solutions.

To emphasize the issues we reiterate the test given in [20] and calculate a sine pulse propagation in a half plane with a constant mean flow of Mach 0.5 in xdirection. The domain is discretized with a grid consisting of distorted elements. For excitation, we choose an initial acoustic pressure distribution, with a peak located below the center of the computational domain and an initial velocity distribution equal to $\mathbf{0}$. This setup leads to a pulse propagating through the domain which is convected due to the given mean flow field. In addition, we choose sound hard walls on the domain boundary, i.e. $\mathbf{u} \cdot \mathbf{n}=0$ but stop the computation before the wave reaches the boundary of the computational domain. The results of this direct approach are displayed in Fig. 1. It can

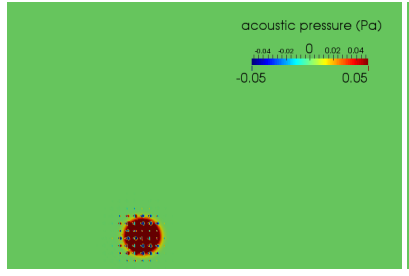

(a) $t=T_{1}$

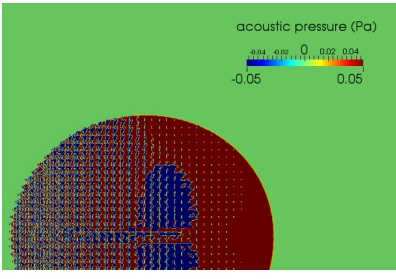

(b) $t=T_{2}$

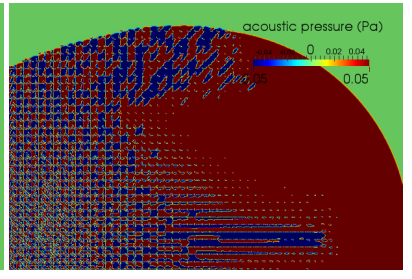

(c) $t=T_{3}$

Figure 1: Wave propagation for initial value problem at different time levels using the direct mixed approach resulting in an exponentially growing solution.

be seen that instabilities occur which propagate through the acoustic domain. Therefore, the straight forward way does not feature a stable numerical scheme as expected. It should be noted, that the same instabilities were observed on an open domain setup approximated by choosing a very large distance to the reflecting boundaries thus indicating a minor impact of the applied boundary conditions. However, it is well known that the convective character of the above equation usually requires an upwinding scheme. In the following, we present a stabilization procedures, and investigate the eigenvalues of the discrete system which gives a good insight to spurious modes and their significance.

\section{Eigenvalues, stabilization and spurious modes}

For the investigation of spurious modes, it is always desirable to fall back to a situation for which physical eigenvalues are analytically given. Such a situation is obtained when assuming a plane, one-dimensional wave propagation in a 
channel of length $L$ and height $H$ with uniform background flow $\mathbf{u}_{0}=\left(u_{0, x}, 0\right)^{t}$. For this case the system of perturbation equations (1) and (2) can be rewritten as

$$
\frac{\partial^{2} p}{\partial t^{2}}+2 M c_{0} \frac{\partial^{2} p}{\partial x \partial t}+\left(M^{2}-1\right) c_{0}^{2} \frac{\partial^{2} p}{\partial x^{2}}=0
$$

in which $M=u_{0, x} / c_{0}$ denotes the Mach number. For a plane wave one can make the ansatz $p(x, t)=\hat{p} e^{j(k x-\omega t)}$ which leads to the characteristic equation

$$
-\omega^{2}+2 \omega M c_{0} k-\left(M^{2}-1\right) c_{0}^{2} k^{2}=0 .
$$

Solving (18) leads to the dispersion relation

$$
k_{ \pm}(\omega)=-\frac{\omega\left(M c_{0} \mp c_{0}\right)}{\left(M^{2} c_{0}^{2}-c_{0}^{2}\right)}=-\frac{\omega}{(M \pm 1) c_{0}} .
$$

and the solution of 17 is obtained by a superposition of $\omega$-dependent plane waves

$$
\hat{p}_{+} e^{j\left(k_{+}(\omega) x-\omega t\right)}+\hat{p}_{-} e^{-j\left(k_{-}(\omega) x-\omega t\right)} .
$$

Considering homogeneous Dirichlet boundary conditions at the extremities of the one dimensional channel, it is possible to show that the admissible frequencies $\omega$ are defined by

$$
\omega_{n}=\frac{c_{0} \pi n}{L}\left(1-M^{2}\right), \quad n \geq 1 .
$$

The discrete eigenvalues for the problem under investigation can be obtained by solving the generalized eigenvalue problem given by

$$
\lambda \mathbf{M x}=\mathbf{K} \mathbf{x},
$$

where $\mathbf{M}$ and $\mathbf{K}$ are defined in 16 . Homogeneous Neumann boundary conditions on the horizontal boundaries and homogeneous Dirichlet on the vertical boundaries are used

$$
\Gamma_{D}=\{0, L\} \times(0, H), \quad \Gamma_{N}=(0, L) \times\{0, H\} .
$$

The first smallest non zero eigenvalues should correspond with the frequencies of the one dimensionnal channel, i.e. $\lambda \simeq j \omega_{n}$ (higher values of $\lambda$ correspond to eigenfunctions oscillating in the height of the channel). We can interpret the discrete eigenvalues as follows:

- If there are small eigenvalues $\lambda$ which do not coincide with the analytical ones, we can say that these are spurious modes.

- If the discrete eigenvalues have a positive real part, the solution becomes unstable.

- Spurious modes with a large negative real part are quickly damped and do not disturb the solution. 


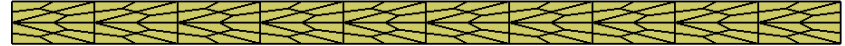

Figure 2: Pseudo one dimensional channel of length $L$ and height $H$, meshed with strongly distorted elements.

So, we distinguish not only between physical and spurious modes but also between evanescent and exponentially growing modes depending on the sign of their real part. For the numerical evaluation of the discrete eigenvalues a long and thin channel with a length to width ratio of $20: 1$ is chosen. The channel is discretized with strongly distorted quadrilateral elements as displayed in Fig. 2 .

Initially the flow velocity is set to zero. So basically, the eigenvalues for the mixed variational formulation of the conservation equations of linear acoustics are evaluated. The result is displayed in Fig. 3 One can see the analytical eigenvalues as the solid lines and the discrete ones indicated by the markers. All discrete eigenvalues have zero real part and coincide with the analytical ones. In

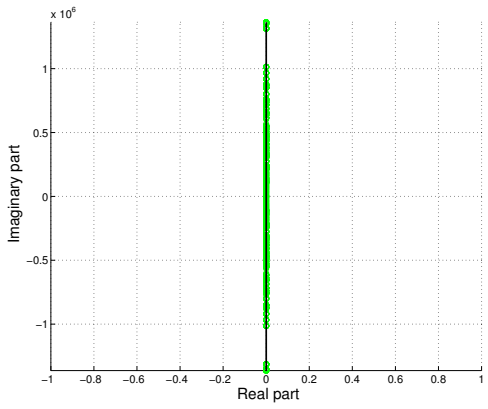

(a) Eigenvalues in the complex plane.

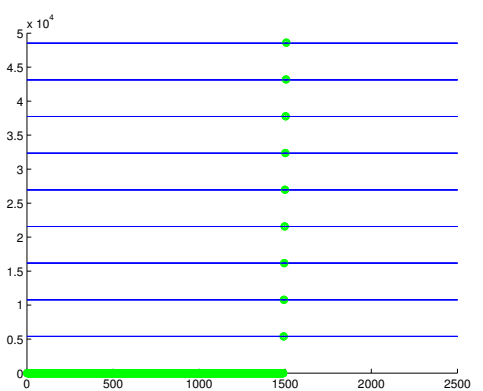

(b) Analytical (blue lines) and absolute value of discrete eigenvalues.

Figure 3: Discrete and analytic eigenvalues. Color coded for high negative real part (blue $*$ ), close to imaginary axis with negative real part and absolute values less or equal to $10^{-7}$ (green $\circ$ ).

contrast to the stable computations with zero Mach number the situation looks different when a constant mean flow of Mach 0.1 is added. As shown in Fig. 4 . a lot of spurious modes are added to the system. Even more dramatically, spurious modes with positive real part occur, which are responsible for the unstable computations already observed. So, it is obvious that one needs to transform the discrete system to ensure stable results. At every step during the stabilization procedure, the discrete eigenvalues will be evaluated to directly show the effects on the numerical scheme.

\subsection{Introduction of numerical flux}

It has already been pointed out, that the choice of $\mathbf{u} \in\left(L^{2}\right)^{d}$ is in contradiction to the convective term in (13), which is assumed to have a significant 


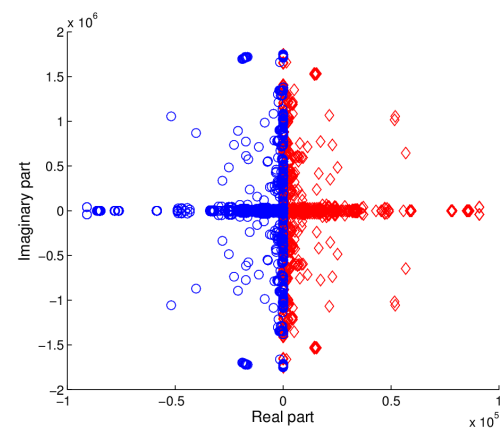

(a) Eigenvalues in the complex plane.

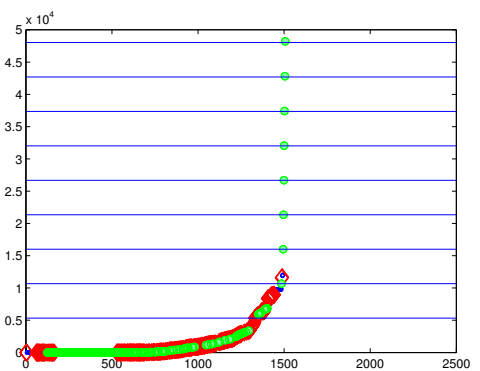

(b) Analytical (blue lines) and absolute value of discrete eigenvalues.

Figure 4: Discrete and analytic eigenvalues for Mach 0.1. Color coded for high negative real part (blue $*$ ), close to imaginary axis with negative real part and absolute values less or equal to $10^{-7}$ (green $\circ$ ) and with positive real part(red).

impact on the stability. To cope with this issue, ideas from DG methods are adopted.

Assume two elements $K_{1}$ and $K_{2}$ with a common boundary $\Gamma_{12}$ as displayed in Fig. 5. For the $\mathrm{x}$-component of the vector quantity $\mathbf{u}$ we can define the average as

$$
\left\{\left\{u_{x}\right\}\right\}=\frac{1}{2}\left(u_{x, 1}+u_{x, 2}\right),
$$

in which $u_{x, 1}$ is the $\mathrm{x}$-component ${ }^{1}$ of the particle velocity associated with the element $K_{1}$ and $u_{x, 2}$ the corresponding unknown associated with the element $K_{2}$. Accordingly we define the jump between the elements as

$$
\left[\left[u_{x}\right]\right]=u_{x, 1} \mathbf{n}_{1}+u_{x, 2} \mathbf{n}_{2},
$$

where $\mathbf{n}_{1}$ and $\mathbf{n}_{2}$ denote the outward normal with respect to the element $K_{1}$ and $K_{2}$, respectively.

In order to introduce a numerical flux for the convective bilinear form instead of (13), we integrate over each element and integrate by part the convective terms

$$
\begin{aligned}
\int_{K_{i}}\left(\mathbf{u}_{0} \cdot \nabla\right) \mathbf{u} \cdot \boldsymbol{\psi} \mathrm{d} K= & -\int_{K_{i}} \mathbf{u} \cdot\left(\mathbf{u}_{0} \cdot \nabla\right) \boldsymbol{\psi} \mathrm{d} K-\underbrace{\int_{K_{i}} \mathbf{u}\left(\nabla \cdot \mathbf{u}_{0}\right) \cdot \boldsymbol{\psi} \mathrm{d} K}_{=0} \\
& +\int_{\partial K_{i}}\left(\mathbf{n} \cdot \mathbf{u}_{0}\right) \mathbf{u} \cdot \boldsymbol{\psi} \mathrm{d} \Gamma .
\end{aligned}
$$

\footnotetext{
${ }^{1}$ The same definition holds for the y-component of the vector field (or the z-component if a three dimensional setting is considered).
} 


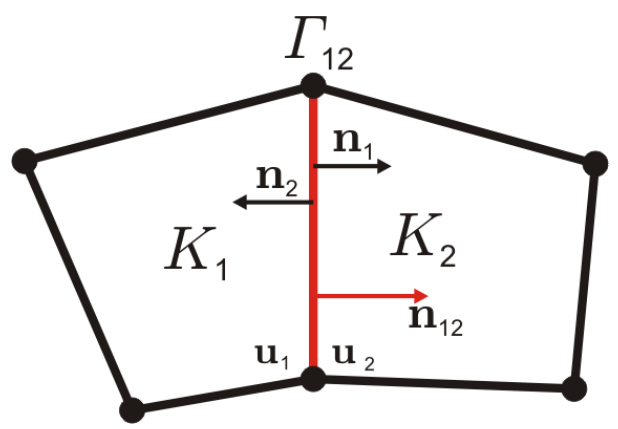

Figure 5: Two elements with common boundary and element local normal vectors as well as the unique normal vector $\mathbf{n}_{12}$ associate to the edge $\Gamma_{12}$.

Although $\nabla \cdot \mathbf{u}_{0}$ is zero due to the assumption of spatially constant flow (or diveregnce free flow), it has to be noted that the formulation in general also allows element-wise constant mean flow functions.

Due to the discontinuous choice for the unknowns and test functions, the boundary term does not necessarily vanish between two interior elements $K_{i}$ and $K_{j}$. A flux term is then introduced on the boundary $\Gamma_{i j}$. Due to the structure of the convective term it is now possible to write the flux for each component of $\mathbf{u}$ individually. The flux across the edge $\Gamma_{12}$ can then be defined for the $2 \mathrm{D}$ problem as

$$
a_{12}(\mathbf{u}, \boldsymbol{\psi})=\int_{\Gamma_{12}}\left\{\left\{\mathbf{u}_{0} u_{x}\right\}\right\} \cdot\left[\left[\psi_{x}\right]\right] \mathrm{d} \Gamma+\int_{\Gamma_{12}}\left\{\left\{\mathbf{u}_{0} u_{y}\right\}\right\} \cdot\left[\left[\psi_{y}\right]\right] \mathrm{d} \Gamma .
$$

The extension to the three dimensional case is straight forward. The bilinear form including numerical fluxes reads as

$$
B=-\sum_{K_{i}} \int_{K_{i}} \mathbf{u} \cdot\left(\mathbf{u}_{0} \cdot \nabla\right) \boldsymbol{\psi} \mathrm{d} K+\sum_{\Gamma_{i j}} a_{i j}(\mathbf{u}, \boldsymbol{\psi})+\int_{\Gamma}\left(\mathbf{n} \cdot \mathbf{u}_{0}\right) \mathbf{u} \cdot \boldsymbol{\psi} \mathrm{d} \Gamma
$$

this equality being now true for discontinuous functions $(\mathbf{u}, \boldsymbol{\psi}) \in V_{h}^{k} \times V_{h}^{k}$. Note that the boundary term along $\Gamma$ vanishes because of the boundary condition (14).

\subsection{Skew symmetry property}

Even with the introduction of the flux term, the stiffness matrix is not obviously skew symmetric, which is desirable due to energy conservation and stability issues as pointed out e.g. in [23]. In order to prove skew symmetry, we 
perform another integration by parts on the right hand side of $(27)$ and obtain

$$
\begin{aligned}
-\sum_{K_{i}} \int_{K_{i}} \mathbf{u} \cdot\left(\mathbf{u}_{0} \cdot \nabla\right) \boldsymbol{\psi} \mathrm{d} K+\sum_{\Gamma_{i j}} a_{i j}(\mathbf{u}, \boldsymbol{\psi})=\sum_{K_{i}} \int_{K_{i}}\left(\mathbf{u}_{0} \cdot \nabla\right) \mathbf{u} \cdot \boldsymbol{\psi} \mathrm{d} K \\
\quad-\sum_{\Gamma_{i j}} \int_{\Gamma_{i j}}\left(\mathbf{n}_{i} \cdot \mathbf{u}_{0}\right) \mathbf{u}_{i} \cdot \boldsymbol{\psi}_{i}+\left(\mathbf{n}_{j} \cdot \mathbf{u}_{0}\right) \mathbf{u}_{j} \cdot \boldsymbol{\psi}_{j} \mathrm{~d} \Gamma \\
+\sum_{\Gamma_{i j}} a_{i j}(\mathbf{u}, \boldsymbol{\psi}) .
\end{aligned}
$$

Averaging of the right hand sides of 27] and 28 yields the final bilinear form

$$
\begin{aligned}
B & =\frac{1}{2} \sum_{K_{i}}(\underbrace{\int_{K_{i}}\left(\mathbf{u}_{0} \cdot \nabla\right) \mathbf{u} \cdot \boldsymbol{\psi} \mathrm{d} K}_{B_{1}}-\underbrace{\int_{K_{i}} \mathbf{u} \cdot\left(\mathbf{u}_{0} \cdot \nabla\right) \boldsymbol{\psi} \mathrm{d} K}_{B_{2}}) \\
& -\frac{1}{2} \sum_{\Gamma_{i j}}\left(\int_{\Gamma_{i j}}\left(\mathbf{n}_{i} \cdot \mathbf{u}_{0}\right) \mathbf{u}_{i} \cdot \boldsymbol{\psi}_{j} \mathrm{~d} \Gamma+\int_{\Gamma_{i j}}\left(\mathbf{n}_{j} \cdot \mathbf{u}_{0}\right) \mathbf{u}_{j} \cdot \boldsymbol{\psi}_{i} \mathrm{~d} \Gamma\right) .
\end{aligned}
$$

Compared to (15), the variational terms are defined with less assumptions on the regularity of the test functions and are therefore compatible with our formulation. Note also that the matrix entries of this bilinear form are skew symmetric. This becomes apparent when one thinks about the direction of the element normals $\left(\mathbf{n}_{i}=-\mathbf{n}_{j}\right)$. Furthermore, it has to be notice that computing the difference $B_{1}-B_{2}$ is simpler than computing each terms separately. With $(\mathbf{u}, \boldsymbol{\psi}) \in V_{h}^{k} \times V_{h}^{k}$, we obtain

$$
\begin{aligned}
B_{1} & =\sum_{K_{i}} \int_{\hat{K}}\left(\mathbf{u}_{0} \cdot \mathcal{J}_{i}^{-T} \hat{\nabla}\right)\left(\frac{\mathcal{J}_{i}}{\left|\mathcal{J}_{i}\right|} \hat{\mathbf{u}}\right) \cdot \mathcal{J}_{i} \hat{\boldsymbol{\psi}} \mathrm{d} \hat{K} \\
B_{2} & =\sum_{K_{i}} \int_{\hat{K}_{i}} \mathcal{J}_{i} \hat{\mathbf{u}} \cdot\left(\mathbf{u}_{0} \cdot \mathcal{J}_{i}^{-T} \hat{\nabla}\right)\left(\frac{\mathcal{J}_{i}}{\left|\mathcal{J}_{i}\right|} \hat{\boldsymbol{\psi}}\right) \mathrm{d} \hat{K},
\end{aligned}
$$

and the computation of $B_{1}-B_{2}$ results in

$$
\sum_{K_{i}}\left(\int_{\hat{K}} \mathcal{J}_{i} \hat{\boldsymbol{\psi}} \cdot \frac{\mathcal{J}_{i}}{\left|\mathcal{J}_{i}\right|}\left(\mathbf{u}_{0} \cdot \mathcal{J}_{i}^{-T} \hat{\nabla}\right) \hat{\mathbf{u}} \mathrm{d} \hat{K}-\int_{\hat{K}_{i}} \mathcal{J}_{i} \hat{\mathbf{u}} \cdot \frac{\mathcal{J}_{i}}{\left|\mathcal{J}_{i}\right|}\left(\mathbf{u}_{0} \cdot \mathcal{J}_{i}^{-T} \hat{\nabla}\right) \hat{\boldsymbol{\psi}} \mathrm{d} \hat{K}\right)
$$

meaning that derivatives of the Jacobian matrices $\mathcal{J}_{i}$ need not to be computed.

Finally, we consider the convective term in (1) with $\rho_{0} c_{0}^{2}=1$. Its variational form reads as

$$
\int_{\Omega} \frac{\partial p}{\partial t} \varphi \mathrm{d} \Omega+\int_{\Omega}(\nabla \cdot \mathbf{u}) \varphi \mathrm{d} \Omega+\int_{\Omega}\left(\mathbf{u}_{0} \cdot \nabla p\right) \varphi \mathrm{d} \Omega=0 .
$$


After applying Green's formula, it is equivalent to

$$
\begin{aligned}
\int_{\Omega} \frac{\partial p}{\partial t} \varphi \mathrm{d} \Omega & -\int_{\Omega} \mathbf{u} \cdot \nabla \varphi \mathrm{d} \Omega+\frac{1}{2} \int_{\Omega}\left(\mathbf{u}_{0} \cdot \nabla p\right) \varphi \mathrm{d} \Omega \\
& -\frac{1}{2} \int_{\Omega}\left(\mathbf{u}_{0} \cdot \nabla \varphi\right) p \mathrm{~d} \Omega-\frac{1}{2} \int_{\Omega}\left(\nabla \cdot \mathbf{u}_{0}\right) p \varphi \mathrm{d} \Omega \\
& +\frac{1}{2} \int_{\Gamma} p \varphi \mathbf{u}_{0} \cdot \mathbf{n} \mathrm{d} \Gamma+\int_{\Gamma} \varphi \mathbf{u} \cdot \mathbf{n} \mathbf{d} \Gamma=0 .
\end{aligned}
$$

Assuming that constant or more generally divergence free flows are used, and applying boundary conditions (14), we may rewrite (31) as

$$
\begin{aligned}
\int_{\Omega} \frac{\partial p}{\partial t} \varphi \mathrm{d} \Omega & -\int_{\Omega} \mathbf{u} \cdot \nabla \varphi \mathrm{d} \Omega \\
& +\frac{1}{2} \int_{\Omega}\left(\mathbf{u}_{0} \cdot \nabla p\right) \varphi \mathrm{d} \Omega-\frac{1}{2} \int_{\Omega}\left(\mathbf{u}_{0} \cdot \nabla \varphi\right) p \mathrm{~d} \Omega=0,
\end{aligned}
$$

which proves the skew-symmetry property of $\mathbf{C}_{\mathbf{p}}$ under the adequate boundary conditions (14). Modifying the original variational form according to 32 and $(29)$ guarantees skew symmetry for the algebraic system of equations and thus energy conservation.

Turning back to the one-dimensional channel example the eigenvalue analysis is started again and one obtains the discrete eigenvalues displayed in Fig.6. Due

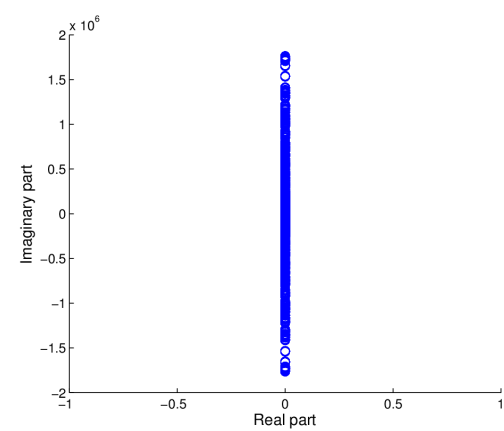

(a) Eigenvalues in the complex plane.

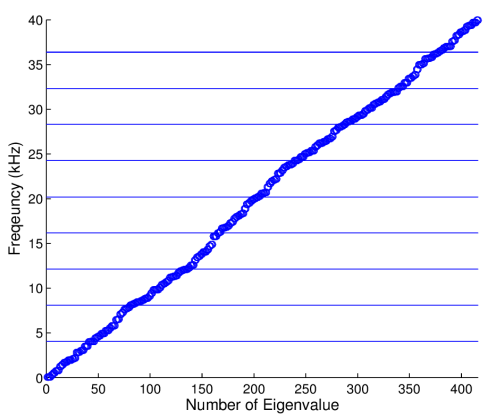

(b) Analytical (blue lines) and absolute value of discrete eigenvalues.

Figure 6: Discrete and analytic eigenvalues for Mach 0.1. All eigenvalues have zero real part but a lot of spurious modes arise in addition to the physical ones.

to the new formulation of the discrete system, the eigenvalues are now located on the imaginary axis. Nevertheless, the system shows a lot of non-physical modes, which can pollute the solution of the system. To illustrate this behaviour, we compute the wave propagation of a pulse in a uniform mean flow of Mach 0.5 as depicted for a characteristic time step in Fig. 7. The basic wave pattern is captured and also the reflection at the lower wall is physical. We used the 


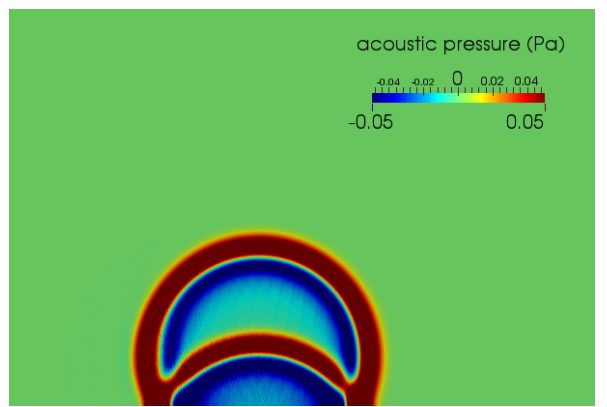

(a) $t=T_{2}$

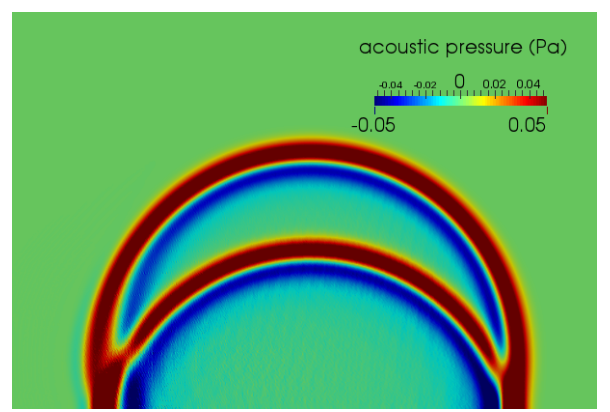

(b) $t=T_{3}$

Figure 7: Contours of pulse wave propagation in homogeneous mean flow of Mach 0.5. Stable computation but spurious waves visible.

same computational grid with linear finite elements as in Fig. 1 and observe non-physical waves.

To increase the convergence of the numerical scheme we follow the approach introduced in [10] for a full DG scheme. It was shown, that spurious waves can be suppressed if a penalization term between the discontinuous elements was added to the system. Additionally, those terms can be seen as an upwinding method for DG as, e.g., used in [8. In the following section, we will therefore incorporate a similar term to the variational form of the momentum conservation (see (2)) to increase the convergence of the method.

\subsection{Upwinding via penalization}

Upwinding is a well known technique to stabilize the numerical solution of differential equations. In many DG methods approximate Rieman solvers are utilized to stabilize a convective equation with jumps 8 .

Due to the different structure of the convective terms in the case under investigation one can define the upwind in a more intuitive way with respect to the unique edge normal $\mathbf{n}_{12}$. Such an upwind flux is usually (written here only for the x-component)

$$
\left.\left\{\begin{array}{ll}
\left\{\mathbf{u}_{0}\right. & u_{x}
\end{array}\right\}\right\}_{\mathbf{u}}= \begin{cases}\mathbf{u}_{0} u_{x, 1} & \text { if } \mathbf{u}_{0} \cdot \mathbf{n}_{12}>0 \\
\mathbf{u}_{0} u_{x, 2} & \text { if } \mathbf{u}_{0} \cdot \mathbf{n}_{12}<0 \\
\left\{\left\{\mathbf{u}_{0} u_{x}\right\}\right\} & \text { if } \mathbf{u}_{0} \cdot \mathbf{n}_{12}=0\end{cases}
$$

As stated in 24] one can alternatively introduce a penalization term between two adjacent elements which is equivalent to (33). Such a penalization was also used in [10] to remove spurious waves in the context of a full DG formulation. Indeed, one can show that

$$
\left\{\left\{\mathbf{u}_{\mathbf{0}} u_{x}\right\}\right\}_{\mathbf{u}} \cdot \mathbf{n}_{12}=\left(\left\{\left\{\mathbf{u}_{\mathbf{0}} u_{x}\right\}\right\}+\alpha\left[\left[u_{x}\right]\right]\right) \cdot \mathbf{n}_{12} .
$$


Here, the parameter $\alpha$ denotes the upwinding parameter. By setting

$$
\alpha=\frac{\left|\mathbf{u}_{\mathbf{0}} \cdot \mathbf{n}\right|}{2}
$$

the penalization is exactly equivalent to $(33)$. The penalization is applied to the convective bilinear form which yields the transformed formulation of the initial bilinear form as

$$
\begin{aligned}
\int_{\Omega}\left(\mathbf{u}_{\mathbf{0}} \cdot \nabla\right) \mathbf{u}^{\mathbf{a}} \cdot \boldsymbol{\psi} \mathrm{d} \Omega \rightarrow & \sum_{\forall K_{i}} \frac{1}{2}\left(\int_{K_{i}}\left(\mathbf{u}_{\mathbf{0}} \cdot \nabla\right) \mathbf{u} \cdot \boldsymbol{\psi} \mathrm{d} K-\int_{K_{i}} \mathbf{u} \cdot\left(\mathbf{u}_{\mathbf{0}} \cdot \nabla\right) \boldsymbol{\psi} \mathrm{d} K\right) \\
& -\sum_{\forall \Gamma_{i j}} \frac{1}{2}\left(\int_{\Gamma_{i j}}\left(\left(\mathbf{u}_{\mathbf{0}} \cdot \mathbf{n}_{j}\right) u_{x, j} \psi_{x, i}+\left(\mathbf{u}_{\mathbf{0}} \cdot \mathbf{n}_{i}\right) u_{x, i} \psi_{x, j}\right) \mathrm{d} \Gamma\right. \\
& \left.+\int_{\Gamma_{i j}} \alpha\left[\left[u_{x}\right]\right] \cdot\left[\left[\psi_{x}\right]\right] \mathrm{d} \Gamma+\ldots\right) .
\end{aligned}
$$

Turning one last time to the eigenvalues of the discrete system as depicted in Fig. 8, we observe that all spurious modes were shifted to the negative half-plane which cause them to decay rapidly.

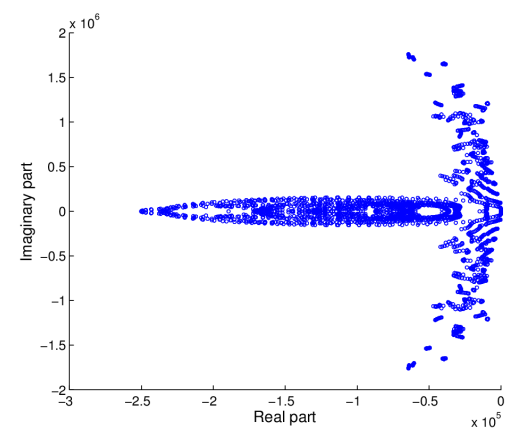

(a) Eigenvalues in the complex plane.

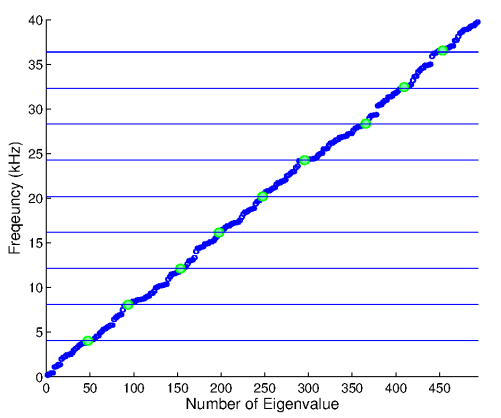

(b) Analytical (blue lines) and absolute value of discrete eigenvalues.

Figure 8: Discrete and analytic eigenvalues for Mach 0.1. Color coded for high negative real part (blue $*$ ), close to imaginary axis with negative real part and absolute value less or equal to $10^{-7}$ (green $\circ$ ).

In fact, the benefits of the penalization term are significant as pictured in Fig. 9 showing the initial two dimensional example. The instabilities of the initial formulation and the non-physical waves are completely removed.

Even though, the complete stabilization procedure was derived for a constant mean flow field, it also covers formulations based on all incompressible flows for which $\nabla \cdot \mathbf{u}_{\mathbf{0}}=0$ is fulfilled. 


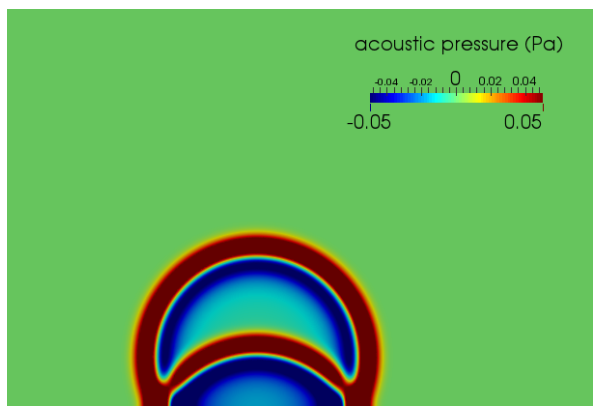

(a) $t=T_{2}$

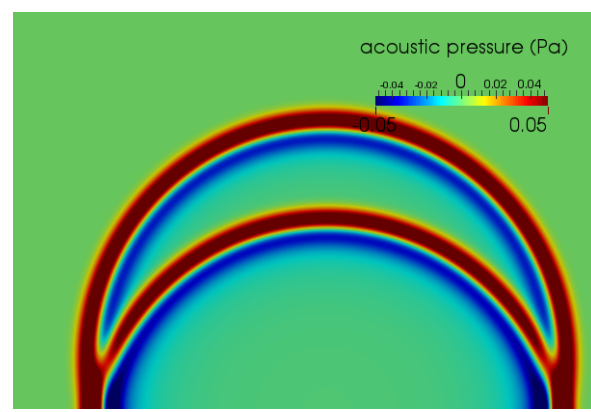

(b) $t=T_{3}$

Figure 9: Contours of pulse wave propagation in homogeneous mean flow of Mach 0.5. No instabilities and no spurious waves.

\section{Numerical results}

\subsection{Convergence analysis}

For a more detailed investigation of the convergence properties of the proposed stabilization procedure, we investigate the benchmark problem depicted in Fig. 10a. According to 25, using as initial conditions

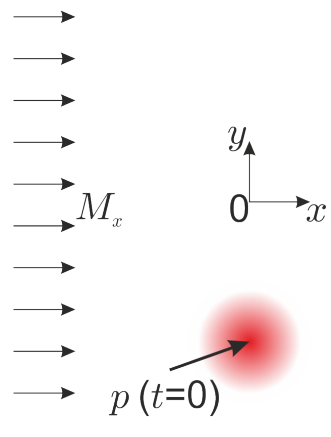

\section{Solid wall}

(a) Computational setup for convergence analysis.

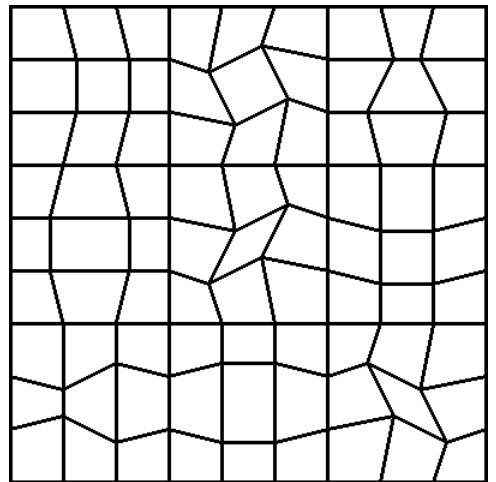

(b) Unit-cell of discretization.

Figure 10: Computational domain and the distorted discrtization pattern.

$$
\mathbf{u}(\mathbf{x}, 0)=\mathbf{0} ; \quad p(x, y, t=0)=\exp \left(\ln (2) \frac{x^{2}+(y+75)^{2}}{25}\right)
$$

the analytical solution for the acoustic pressure can be given as

$$
p(t, x, y)=\frac{1}{2 \mu} \int_{0}^{\infty} e^{-\frac{\zeta^{2}}{4 \mu}} \cos (\xi t)\left[J_{0}(\xi \eta)+J_{0}(\xi \zeta)\right] \xi d \xi,
$$


in which $J_{0}$ denotes the Bessel function of first kind, along with the abbreviations

$$
\begin{aligned}
\mu & =\frac{\ln 2}{25}, \\
\eta & =\sqrt{\left(x-M_{x} t\right)^{2}+(y-25)^{2}} \\
\zeta & =\sqrt{\left(x-M_{x} t\right)^{2}+(y+25)^{2}} .
\end{aligned}
$$

In all computations, we have chosen a uniform flow of Mach 0.5.

The convergence is analyzed on a distorted computational grid, built from the unit-cell depicted in Fig. 10b. In this pattern, almost all possible deformations of a quadrilateral cell are covered. To obtain $h$ refinements, the pattern is scaled by the given factor. The solid boundary is modeled using homogeneous Neumann boundary conditions. To cover the free field radiation, we compute the solution in a very large computational domain and terminate the calculation before the wave reaches the boundary. For a time discretization we utilize a fourth order Runge Kutta scheme and compute the time-step as one tenth of the CFL-number w.r.t. the finest grid. The analysis is conducted for three refinement levels in $h$ and $k$, resulting in 12 computations. A limiting factor was the accuracy of evaluating the indefinite integral in (36) in each integration point for the reference solution. This has been done using an adaptive integration procedure provided by the boost library for $\mathrm{C}++$. The numerical errors for

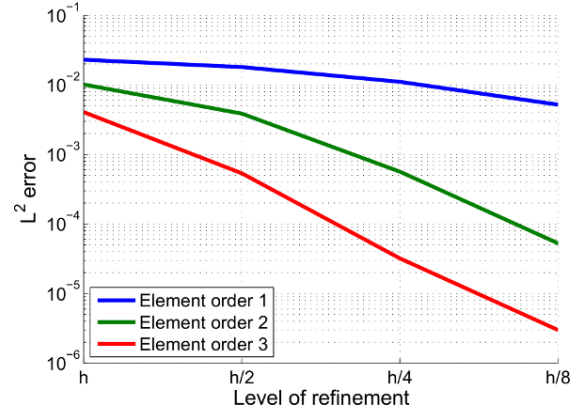

(a) Convergence in the $L^{2}$ norm.

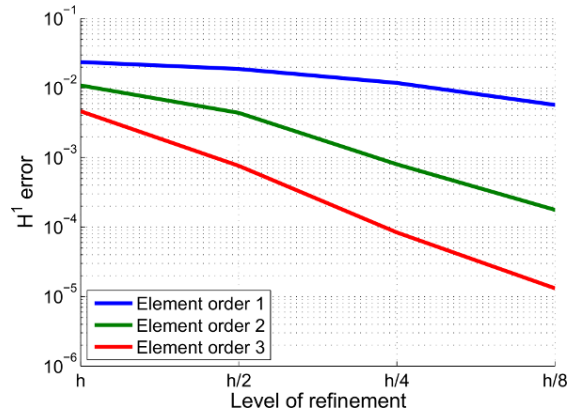

(b) Convergence in the $H^{1}$ norm.

Figure 11: Convergence of computational scheme $h$ and $k$ refinement.

the presusre are computed in the $L^{2}$ and $H^{1}$ norm, and are displayed in Fig. 11 . Thereby, the computations have been performed for a single time-step at the end of the simulation run and integrated over the computational domain. For all computations, we observe convergence with the expected order. The rates are explicitly given for the $H^{1}$-norm in Tab. 1 and show expected values.

For all computations we utilized a penalization factor $\alpha=\alpha_{0}\left|\mathbf{u}_{\mathbf{0}} \cdot \mathbf{n}\right|$ with $\alpha_{0}=0.5$. In a second step, we investigate the behavior of the computational scheme for different values of $\alpha_{0}$, and obtained the results as depicted in Fig. 12 . We observe the smallest numerical error for the proposed value of $\alpha_{0}=0.5$. 


\begin{tabular}{c|cccc}
$p \backslash h$ & $h \rightarrow h / 2$ & $h / 2 \rightarrow h / 4$ & $h / 4 \rightarrow h / 8$ & Average \\
\hline 1 & 0.2916 & 0.6146 & 1.0094 & 0.8126 \\
2 & 1.2489 & 2.3958 & 2.3516 & 1.8632 \\
3 & 2.5485 & 3.2970 & 2.7920 & 2.8791
\end{tabular}

Table 1: Convergence rates in the $H^{1}$ norm

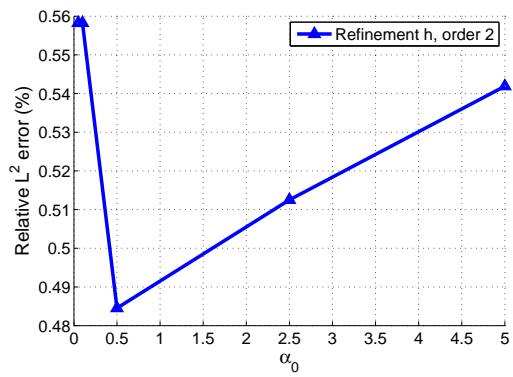

(a) $L^{2}$ error for varying alpha.

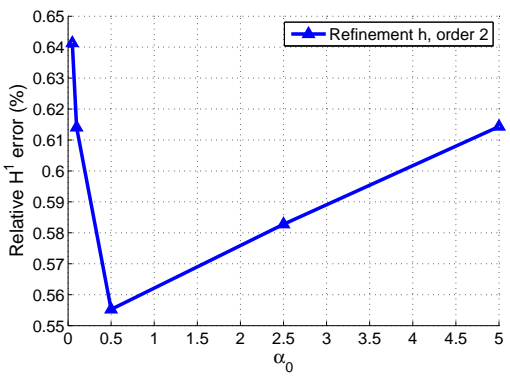

(b) $H^{1}$ error for varying alpha.

Figure 12: Computational error for different values of $\alpha$ for second $h$ refinement level and second order elements.

Choosing higher or lower values leads to higher numerical errors or even instabilities in the solutions (certainly due to the violation of the time step restriction).

Those results hold also for higher element orders. By using the adaptive visualization capabilities of the software gmsh [26], it is possible to display the result of a seventh order computation as displayed in Fig. 13 Given in the solid black lines is the computational grid. It can be seen, that this initial discretization resolves the pulse with just two elements which is compensated by increasing the element order. Due to convection the wave form is displaced from the center, it is not distorted and no spurious waves are visible.

\subsection{Aeroacoustic computation of human phonation process}

The numerical simulation of the human phonation process is a complex task involving the accurate computation of the flow field interacting with the mechanics of the vocal folds and the induced acoustic field. Fortunately, the fully coupled scheme does not need to be computed and some simplifications can be made. First of all, one can safely assume an incompressible flow due to the low flow velocity inside the larynx thus reducing complexity and motivating a hybrid approach towards the aeroacoustic components of the sound field. Secondly, vibroacoustic components are of minor importance which was shown in [27. Therefore the paper in hand focuses on the aeroacoustic field computation.

The basic setup under investigation is depicted in Fig. 14 including the flow and acoustic grid. For the acoustic grid an average element edge length of 


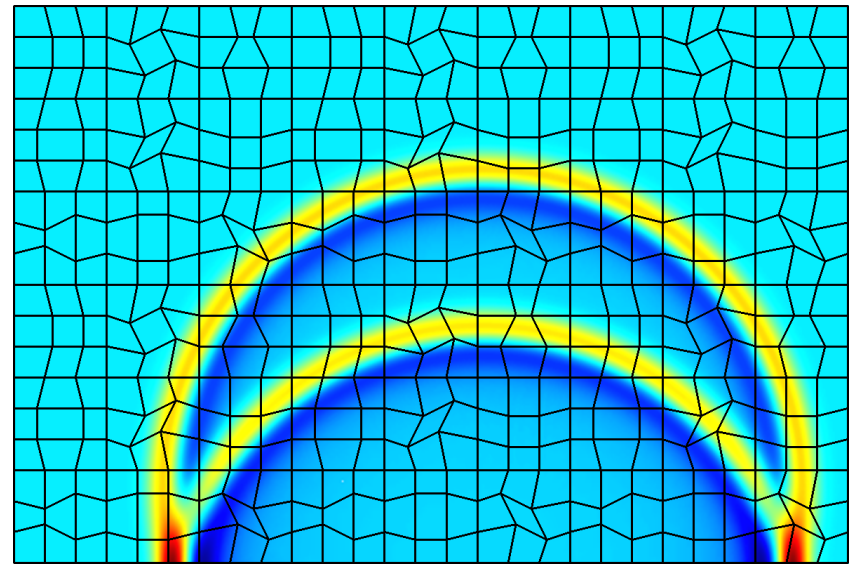

Figure 13: Contours of acoustic pressure at the end of the simulation run for coarsest grid, 7 th order elements and utilization of adapative visualization in $g m s h$.

$h_{e} \approx 0.5 \mathrm{~mm}$ is chosen. Thereby the acoustic solution is computed on only 20000 second order elements. The resulting computational grid is capable to resolve frequencies up to $100 \mathrm{kHz}$ with approximately 6 elements per wavelength and is thereby more than sufficient to resolve the frequency range of interest $(10-5000 \mathrm{~Hz})$. A time step size of $\Delta t=1 \mathrm{~ms}$ is chosen for the fourth order Runge Kutta time stepping scheme. At the boundaries of the acoustic domain, we model an infinitely long channel by an application of a time domain perfectly matched layer (PML) as given in 28].

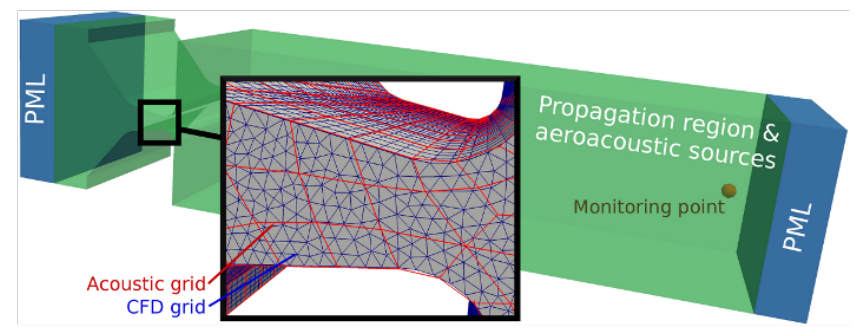

Figure 14: Computational setup for the three dimensional case including the location of the monitoring point. Magnification of CFD and CAA grids inside the glottis. Remark: The $P M L$ are purely acoustic PML (the mean flow vanishes at their boundary, see Fig. 15).

The flow domain is discretized using 2.3 million wedge shaped cells. No-slip boundary conditions are imposed on the walls of the channel as well as on the surface of the vocal folds. At the left hand side of the channel, a pressure inlet at $300 \mathrm{~Pa}$ is chosen which results in an maximum mean flow velocity of $44 \mathrm{~m} / \mathrm{s}$ as depicted in Fig. 15. It is visible, that the field varies significantly through the domain, which is why we include all terms of the perturbation equation in our 
computation. Additionally, the highest flow velocities are observed inside the glottis. On the right hand side a pressure outlet boundary condition is chosen, setting the fluid pressure to zero.

To avoid the full three dimensional fluid structure interaction, the vocal folds are set in motion by a prescribed periodic displacement. By this boundary condition, the glottis, i.e. the region between the vocal folds, is opened and closed with a frequency of $100 \mathrm{~Hz}$. These cycles induce a modulatin of the flow passing through the glottis thus inducing sound generating sources. Therefore, the prescribed frequency of motion is also expected to appear in the recorded acoustic pressure signals. For a detailed discussion on the flow computation, we refer to [29.

For the analysis, the transient start-up phase is ignored and only the last 1900 time steps are used. Based on these timesteps the mean flow field is

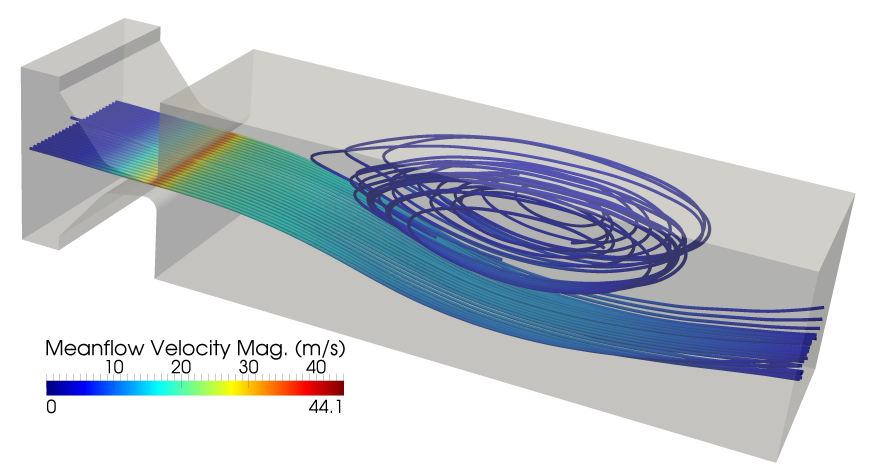

Figure 15: Streamlines of mean flow velocity. Asymmetric structure and large scale vortex.

computed as displayed in Fig. 15. The behaviour is due to the tendency of the flow in the supra-glottal region to attach to one side of the trachea more often than to the other. Also visible in the steam-lines is a vortex in the mean flow field at the top of the domain.

Depicted in Fig. 16 are the results for the sound pressure level(SPL) at the end of the simulation domain for a characteristic monitoring point. Most significant is the tonal component at $100 \mathrm{~Hz}$ which corresponds to the frequency of the prescribed vocal fold movement as expected. The higher harmonic peaks are observed to have lower amplitudes and starting from $800 \mathrm{~Hz}$ no tonal component can be observed.

It has to be noted that the computed acoustic field considering a spatially varying mean flow field shows no instability. Additionally, the computational grid used in the acoustic field computation was generated by the extrusion of a free quadrilateral surface mesh. The resulting volume discretization was thereby irregular and not aligned with the mean flow field. This gives good confidence in the robustness of the scheme and its applicability to simulate flow induced sound in the presence of a solenoidal mean flow field. 


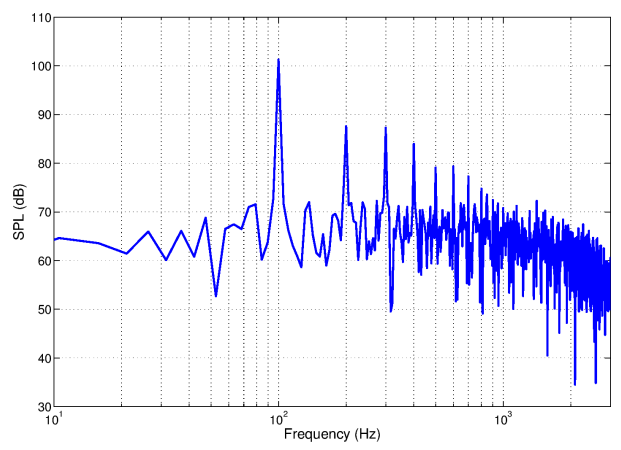

Figure 16: SPL spectrum recorded at the end of simulation domain.

\section{Conclusion}

Within this paper, we presented a stabilized finite element method for hyperbolic systems of partial differential equations including convective effects. The only assumption on the mean flow field made during the derivation was the zero divergence property which holds true in many applications, e.g. in computational aeroacoustics at low Mach numbers. The detailed derivation of the stabilization procedure should give a good insight to the properties of the method in the special context of a mixed finite element method. We could thereby demonstrate that our discrete scheme ensures skew symmetry in the global equation systemeliminates exponentially growing solutions and the penalization term suppresses spurious modes. Thereby, it is possible to compute systems of partial differential equations including convective terms in a stable and consitent manner. Furthermore, it was possible to show, that the order of convergence is not reduced due to the stabilization process and we observe the expected rates for the spectral finite element method. Also, the diagonal mass matrices enable the utilization of explicit time stepping schememes which makes the overall method attractive for optimized implementations. Alternatively, it should be possible to include the method in existing finite element codes with only a minor additional effort. Except for the discontinuous mapping of equation numbers for the velocity unknonws and the evaluation of element fluxes, all terms can be evaluated using standard FE procedures. The final example of an investigation of the process of human phonation shows the applicability of the method to aeroacoustic computations in three space dimensions without any additional restrictions regarding the numerical scheme itself. 
[1] M. J. Lighthill, On sound generated aerodynamically I. General theory, Proceedings of the Royal Society of London 211 (1951) 564-587.

[2] C. Bailly, D. Juvé, Numerical solution of acoustic propagation problems using linearized euler equations, American Institute of Aeronautics and Astronautics 38 (2000) 22-29.

[3] R. Ewert, W. Schröder, Acoustic perturbation equations based on flow decomposition via source filtering, Journal of Computational Physics 188 (2003) 365-398.

[4] J. Seo, Y. Moon, Perturbed compressible equations for aeroacoustic noise prediction at low mach numbers, AIAA Journal 43 (2005) 1716-1724.

[5] C. Munz, M. Dumbser, S. Roller, Linearized acoustic perturbation equations for low Mach number flow with variable density and temperature, Journal of Computational Physics 224 (2007) 352 - 364 .

[6] J. S. Hesthaven, T. Warburton, Nodal discontinuous Galerkin methods, Texts in Applied Mathematics, Springer, 2008.

[7] P. Delorme, P. A. Mazet, C. Peyret, Y. Ventribout, Computational aeroacoustics applications based on a discontinuous galerkin method, Comptes rendus de l'Académie des sciences - Serie IIb - Mécanique/Mechanics 333 (2005) 676-682.

[8] M. Bauer, J. Dierke, R. Ewert, Application of a discontinuous galerkin method to predict airframe noise, in: 15th AIAA/CEAS Aeroacoustics Conference (30th AIAA Aeroacoustics Conference), 2009.

[9] R. Léger, C. Peyret, S. Piperno, Coupled discontinuous galerkin/finite difference solver on hybrid meshes for computational aeroacoustics, AIAA Journal 50 (2012) 338-349.

[10] N. Castel, G. Cohen, M. Duruflé, Application of discontinuous galerkin spectral method on hexahedral elements for aeroacoustic, Journal of Computational Acoustics 17,2 (2009) 175-196.

[11] F. Assous, P. Degond, E. Heintze, J. S. P. A. Raviart, On a finite-element method for solving the three-dimensional Maxwell equations, J. Comput. Phys. 109 (2) (1993) 222-237.

[12] A. S. Bonnet-BenDhia, G. Legendre, E. Luneville, Analyse mathmatique de l'quation de galbrun en coulement uniforme, Comptes Rendus de l'Acadmie des Sciences - Srie IIB - Mcanique 329 (2001) 601-606.

[13] G. Cohen, M. Duruflé, Non spurious spectral-like element methods for Maxwell's equations, J. Comp. Math. 25 (3) (2007) 282-302. 
[14] J. H. Seo, Y. J. Moon, Linearized perturbed compressible equations for low mach number aeroacoustics, Journal of Computational Physics 218 (2006) $702-719$.

[15] F. Brezzi, M. Fortin, Mixed and Hybrid Finite Element Methods, Springer, New York, 1991.

[16] E. Becache, C. Tsogka, P. Joly, An analysis of new mixed finite elements for the approximation of wave propagation problems, SINUM 37 (2000) $1053-1084$.

[17] M. Benzi, G. H. Golub, J. Liesen, Numerical solution of saddle point problems, ACTA NUMERICA 14 (2005) 1-137.

[18] G. Cohen, S. Fauqueux, Mixed finite elements with mass-lumping for the transient wave equation, Journal of Computational Acoustics 8 (2000) 171188.

[19] A. Hüppe, M. Kaltenbacher, Advanced spectral finite element method for computational acoustics in the mid-frequency range, in: Proceedings of the ISMA 2010, 2010.

[20] A. Hüppe, M. Kaltenbacher, Spectral finite elements for computational aeroacoustics using acoustic pertrubation equations, Journal of Computational Acoustics 20 (2).

[21] P. A. Raviart, J. M. Thomas, A mixed finite element method for 2nd order elliptic problems, Mathematical Aspects of the Finite Element Method, Lecture Notes in Mathematics 606 (1977) 292-315.

[22] A. N. Brooks, T. J. R. Hughes, Streamline upwind/petrov-galerkin formulations for convection dominated flows with particular emphasis on the incompressible navier-stokes equations, Computer Methods in Applied Mechanics and Engineering 32 (1982) 199-259. doi:10.1016/0045-7825(82) 90071-8.

[23] Y. Morinishi, Skew-symmetric form of convective terms and fully conservative finite difference schemes for variable density low-mach number flows, Journal of Computational Physics 229 (2010) 276 - 300.

[24] F. Brezzi, L. Marini, E. Suli, Discontinuous Galerkin methods for firstorder hyperbolic problems, Mathematical Models and Methods in Applied Sciences 14 (2004) $1893-1903$.

[25] C. K. Tam, Benchmark problems and solutions, in: ICASE/LaRC Workshop on Benchmark Problems in Computational Aeroacoustics, 1995.

[26] C. Geuzaine, J.-F. Remacle, Gmsh Reference Manual, http://www.geuz.org/gmsh, 1st Edition (Aug. 2003). 
[27] S. Zörner, M. Kaltenbacher, Fluid-structure-acoustic interaction algorithms and implementations using the finite element method, in: Eccomas $2010,2010$.

[28] A. Hüppe, M. Kaltenbacher, Stable matched layer for the conservation equations of acoustics in the time domain, Journal of Computational Acoustics 20 (1).

[29] P. Šidlof, J. Horáček, V. Řidký, Parallel CFD simulation of flow in a 3d model of vibrating human vocal folds, Computers \& Fluids (0), article in press. doi:10.1016/j.compfluid.2012.02.005. 